\title{
GLOBAL ATTRACTIVITY OF A CLASS OF DELAY DIFFERENTIAL EQUATIONS WITH IMPULSES
}

\author{
YUJI LIU' ${ }^{\prime 2}$ and BINGGEN ZHANG ${ }^{3}$
}

(Received 21 June, 2001; revised 8 September, 2002)

\begin{abstract}
In this paper, we study the global attractivity of the zero solution of a particular impulsive delay differential equation. Some sufficient conditions that guarantee every solution of the equation converges to zero are obtained.
\end{abstract}

\section{Introduction}

Recently, with the rapid development of the theory and applications of impulsive differential equations, the study of the impulsive delay differential equation has attracted the interest of many mathematicians [1-9]. The purpose of this paper is to study the global attractivity of the following impulsive delay differential equation:

$$
\begin{cases}x^{\prime}(t)+a(t) x(t)=p(t)\left(1-e^{x(t-\tau)}\right), & t \geq 0, t \neq t_{k}, \\ x\left(t_{k}^{+}\right)-x\left(t_{k}\right)=b_{k} x\left(t_{k}\right), & k \in N,\end{cases}
$$

where $a(t), p(t) \in C([0,+\infty),[0,+\infty)), \tau>0, b_{k}>-1, p(t)>0$, for all $k \in N, t \geq 0,0<t_{1}<t_{2}<\cdots$, with $t_{k} \rightarrow+\infty$ as $k \rightarrow+\infty$.

In the special case where $p(t)=a N_{0} a(t),(1.1)$ has been used to model the impulsive growth of red blood cells.

As usual, we say that $x(t)$ defined in $[-\tau,+\infty)$ is a solution of $(1.1)$, if $x(t)$ is absolutely continuous at points $t \neq t_{k}$ and at $t=t_{k}, x\left(t_{k}^{+}\right)$exists, $x(t)$ is left-continuous for $t \geq-\tau$, and satisfies (1.1) for $t \geq 0$.

\footnotetext{
${ }^{1}$ Department of Applied Mathematics, Beijing Institute of Technology, Beijing 100081, P. R. China. ${ }^{2}$ Department of Mathematics, Yueyang Teacher's University, Yueyang, Hunan 414000, P. R. China. ${ }^{3}$ Department of Mathematics, Ocean University of China, Qingdao 266071, P. R. China; e-mail: bgzhang@public.qd.sd.cn.

(C) Australian Mathematical Society 2003, Serial-fee code 1446-1811/03
} 


\section{Main results}

The main results are as follows.

THEOREM 1. Suppose that:

(i) there is a positive number $p$ such that

$$
p(p+1 / 2)<1
$$

(ii) for any $\epsilon>0$, there exists an integer $N$ such that

$$
\prod_{k=n}^{n+m}\left(1+b_{k}\right)<1+\epsilon, \quad n>N, m \geq 0
$$

(iii)

$$
\int_{0}^{+\infty} p(s) e^{\int_{0}^{s} a(u) d u} \prod_{0 \leq t_{k}<s}\left(1+b_{k}\right)^{-1} d s=+\infty
$$

(iv) for sufficiently large t, we have

$$
\int_{t-\tau}^{t} p(s) e^{\int_{t}^{t} a(u) d u} \prod_{t-\tau \leq t_{k}<s}\left(1+b_{k}\right)^{-1} d s \leq p+\frac{1}{2} e^{-\int_{t-\tau}^{t} a(u) d u}
$$

and

$$
a(t) \geq a(t-\tau), \quad t \geq \tau .
$$

Then every solution of (1.1) tends to zero as $t \rightarrow \infty$.

THEOREM 2. Suppose that (2.2), (2.3) and (2.5) hold and for sufficiently large $t$, we have

$$
\int_{t-\tau}^{t} p(s) e^{\int_{t-\tau}^{s} a(u) d u} \prod_{t-\tau \leq t_{k}<s}\left(1+b_{k}\right)^{-1} d s \leq 3 / 2 .
$$

Then every solution of (1.1) tends to zero as $t \rightarrow \infty$.

REMARK 1. Condition (2.2) is not critical; it allows the convergence of

$$
\prod_{k=1}^{+\infty}\left(1+b_{k}\right)
$$

and the possibility that $-1<b_{k} \leq 0$ as special cases. Condition (2.5) allows constant functions, nondecreasing functions and $\tau$-periodic functions as special cases. 
REMARK 2. If the impulsives disappeared and $a(t) \equiv 0$, Theorem 2 is the main result of [4]. If $a(t) \equiv \lambda$, then the conditions of Theorem 2 are

$$
\int_{0}^{+\infty} p(t) e^{\lambda t} d t=+\infty \text { and } \int_{t-\tau}^{t} p(s) e^{(s-t+\tau) \lambda} d s \leq \frac{3}{2}
$$

which improve the conditions in [5].

\section{Proofs of the theorems}

LEMMA 1. Suppose that (2.2) and (2.3) hold. Then every nonoscillatory solution of (1.1) tends to zero as $t \rightarrow \infty$.

PROOF. Without loss of generality, suppose that $x(t)$ is eventually positive. Then there exists $T_{1} \geq 0$ such that $x(t-\tau)>0$ for $t \geq T_{1}, t \neq t_{k}$. Moreover, $x(t)$ is decreasing in $\left(t_{k}, t_{k+1}\right]$ with $t_{k} \geq T_{1}$. Let $\liminf _{t \rightarrow+\infty} x(t)=\alpha$, then $\alpha \geq 0$. We shall prove that $\alpha=0$. Since $x\left(t_{k}\right)$ is the left minimum value of $x(t)$, there exists a subsequence $\left\{x\left(t_{k_{j}}\right)\right\}$ such that $\lim _{j \rightarrow+\infty} x\left(t_{k_{j}}\right)=\alpha$. If $\alpha>0$, choosing $\epsilon>0$ such that $\alpha-\epsilon>0$, again there exists $T>T_{1}$ such that $x(t-\tau)>\alpha-\epsilon$, for $t \geq T$. Then by (1.1), we have

$$
\prod_{T \leq t_{k}<t_{k_{j}}}\left(1+b_{k}\right)^{-1} x\left(t_{k_{j}}\right)-x(T) \leq\left(1-e^{\alpha-\epsilon}\right) \int_{T}^{t_{k_{j}}} p(s) e^{\int_{T}^{s} a(u) d u} \prod_{T \leq t_{k}<s}\left(1+b_{k}\right)^{-1} d s,
$$

which contradicts (2.2) and (2.3), so $\alpha=0$. Now for any $t \geq T$, there exists $t_{k_{j}}$ such that $t_{k_{j}} \leq t<t_{k_{j+1}}$ and $t_{k_{j}}<t_{k_{j}+1}<\cdots<t_{k_{j}+l} \leq t$. Then

$$
\begin{aligned}
0<x(t)<x\left(t_{k_{j}+l}^{+}\right) & =\left(1+b_{k_{j}+l}\right) x\left(t_{k_{j}+l}\right) \leq\left(1+b_{k_{j}+l}\right) x\left(t_{k_{j}+l-1}^{+}\right) \\
& =\left(1+b_{k_{j}+l}\right)\left(1+b_{k_{j}+l-1}\right) x\left(t_{k_{j}+l-1}\right) \leq \cdots \leq \prod_{s=0}^{l}\left(1+b_{k_{j}+s}\right) x\left(t_{k_{j}}\right) .
\end{aligned}
$$

From (2.3), there exists a constant $A>0$, such that $\prod_{s=0}^{l}\left(1+b_{k_{j}+s}\right) \leq A$ for any $l$ and any $k_{j}$. Hence $0<x(t) \leq A x\left(t_{k_{j}}\right)$. Let $t \rightarrow+\infty$. Then we obtain $\lim _{t \rightarrow+\infty} x(t)=0$.

LEMMA 2. Suppose that (2.2), (2.4) or (2.6), and (2.5) hold. Then every oscillatory solution of (1.1) is bounded.

PROOF. From (2.4) and (2.5), or (2.5) and (2.6), we obtain

$$
\int_{t-\tau}^{t} p(s) e^{\int_{1}^{s} a(u) d u} \prod_{s \leq t_{k}<t}\left(1+b_{k}\right) d s \leq M,
$$


where $M$ is a positive constant. First, we shall prove that $x(t)$ is bounded above. By (1.1),

$$
x^{\prime}(t)+a(t) x(t) \leq p(t), \quad t \geq 0, t \neq t_{k} .
$$

Choose any sequence $\left\{c_{n}\right\}$ such that $x\left(c_{n}\right)=0$ and $0<c_{1}<c_{2}<\cdots$, with $\lim \rightarrow+\infty_{n} c_{n}=+\infty, x(t) \geq 0$ for $t \in\left[c_{2 i-1}, c_{2 i}\right]$ and $x(t) \leq 0$ for $t \in\left[c_{2 i}, c_{2 i+1}\right]$. Let

$$
\hat{x}_{i}=\sup _{t \in\left[c_{2 i-1}, c_{2 i}\right]} x(t) \text { and } \tilde{x}_{i}=\inf _{t \in\left[c_{2 i}, c_{2 i+1}\right]} x(t) \text {. }
$$

We shall prove that $\left\{\hat{x}_{i}\right\}$ and $\left\{\tilde{x}_{i}\right\}$ are bounded. First, we prove that $\left\{\hat{x}_{i}\right\}$ is bounded above; there are two cases to consider.

Case 1: $\hat{x}_{i}$ is the maximum value of $x(t)$ in $\left[c_{2 i-1}, c_{2 i}\right]$. Then there exists $c \in$ $\left(c_{2 i-1}, c_{2 i}\right)$ such that $\hat{x}_{i}=x(c)>0, x^{\prime}(c) \geq 0$. By $(1.1), x(c-\tau) \leq 0$, so there exists $\xi \in(c-\tau, c)$ such that $x(\xi)=0$. Integrating (3.1) from $\xi$ to $c$, we obtain

$$
\hat{x}_{i}=x(c) \leq \int_{\xi}^{c} p(t) e^{f_{c}^{t} a(u) d u} \prod_{t \leq t_{k}<c}\left(1+b_{k}\right) d t \leq M .
$$

Case 2: $\hat{x}_{i}$ is not the maximum value of $x(t)$ in $\left[c_{2 i-1}, c_{2 i}\right]$. Then there exists $t_{k+l} \in\left(c_{2 i-1}, c_{2 i}\right)$ such that $\hat{x}_{i}=x\left(t_{k+l}^{+}\right)$. We assume that $c_{2 i-1}<t_{k+1}<\cdots<t_{k+l}$. There are two possible cases to consider.

Subcase 2.1: $x\left(t_{k+l}\right)$ is the left locally maximum value. By Case 1 , we have $x\left(t_{k+l}\right) \leq M$, so $\hat{x}_{i}=x\left(t_{k+l}^{+}\right)=\left(1+b_{k+l}\right) x\left(t_{k+l}\right) \leq\left(1+b_{k+l}\right) M$.

Subcase 2.2: $x\left(t_{k+l}\right)$ is not the left locally maximum value. There are two possible subcases to consider.

Subcase 2.2.1: If $x\left(t_{k+l}^{+}\right)<x\left(t_{k+l}\right)$, then $x(t)$ has maximum value noted by $x(c)$ in $\left(t_{k+l-1}, t_{k+l}\right)$. By Case $1, x(c) \leq M$, so $\hat{x}_{i}=x\left(t_{k+l}^{+}\right)=\left(1+b_{k+l}\right) x\left(t_{k+l}\right) \leq$ $\left(1+b_{k+l}\right) x(c) \leq\left(1+b_{k+l}\right) M$.

Subcase 2.2.2: If $x\left(t_{k+l-1}^{+}\right) \geq x\left(t_{k+l}\right)$, we have two possible cases to consider.

Subcase 2.2.2.1: If $x\left(t_{k+l-1}\right)$ is the left locally maximum value, then, by Case 1 , $x\left(t_{k+l-1}\right) \leq M$. Thus $\hat{x}_{i}=x\left(t_{k+l}^{+}\right)=\left(1+b_{k+l}\right) x\left(t_{k+l}\right) \leq\left(1+b_{k+l}\right)\left(1+b_{k+l-1}\right) M$.

Subcase 2.2.2.2: $x\left(t_{k+l-1}\right)$ is not the left maximum of $x(t)$. Repeating this process, at the end, if $x\left(t_{k+1}\right)$ is the left locally maximum value of $x(t)$, then $x\left(t_{k+1}\right) \leq M$. Therefore

$$
\hat{x}_{i} \leq \cdots \leq \prod_{s=1}^{l}\left(1+b_{k+s}\right) x\left(t_{k+1}\right) \leq \prod_{s=1}^{l}\left(1+b_{k+s}\right) M .
$$

Otherwise, since $x\left(c_{2 i-1}\right)=0, x(t)$ has maximum value noted by $x(c)$ in $\left(c_{2 i-1}, t_{k+1}\right)$. By Case $1, x(c) \leq M$, so

$$
\hat{x}_{i} \leq \prod_{s=1}^{l}\left(1+b_{k+s}\right) x\left(t_{k+1}\right) \leq \prod_{s=1}^{l}\left(1+b_{k+s}\right) x(c) \leq \prod_{s=1}^{l}\left(1+b_{k+s}\right) M .
$$


Then $\hat{x}_{i} \leq A M$, where $A$ is defined in Lemma 1 .

From Cases 1 and 2 , we have $\hat{x}_{i} \leq \max \{M, A M\}=B$. Next we shall prove that $\left\{\tilde{x}_{i}\right\}$ is bounded below. By (1.1), we obtain

$$
x^{\prime}(t)+a(t) x(t) \geq\left(1-e^{B}\right) p(t), \quad t \geq 0, t \neq t_{k} .
$$

Using a similar method to the above, we obtain

$$
\tilde{x}_{i} \geq\left(1-e^{B}\right) M \quad \text { or } \quad \tilde{x}_{i} \geq\left(1-e^{B}\right) A M .
$$

This shows that $\left\{\tilde{x}_{i}\right\}$ is bounded below, and completes the proof of the lemma.

LEMMA 3. Suppose that (2.1), (2.2), (2.4) and (2.5) hold. Then every oscillatory solution of (1.1) tends to zero as $t \rightarrow+\infty$.

Proof. Suppose $x(t)$ is any oscillatory solution of (1.1). By Lemma 2, $x(t)$ is bounded, so let $\lim \sup _{t \rightarrow+\infty} x(t)=v, \liminf _{t \rightarrow+\infty} x(t)=u$, Then $-\infty<u \leq 0 \leq$ $v<+\infty$, and by (2.2), for any $\epsilon>0$, there exists $N$ such that

$$
\prod_{k=n}^{n+m}\left(1+b_{k}\right)<1+\epsilon, \quad n \geq N, m \geq 0 .
$$

Again for this $\epsilon>0$, there exists $T \geq t_{N}$ such that

$$
u_{1}=u-\epsilon<x(t-\tau)<v+\epsilon=v_{1}, \quad t \geq T .
$$

Then (1.1) gives

$$
\begin{array}{ll}
x^{\prime}(t)+a(t) x(t) \leq\left(1-e^{u_{1}}\right) p(t), & t \geq T, \quad t \neq t_{k}, \\
x^{\prime}(t)+a(t) x(t) \geq\left(1-e^{v_{1}}\right) p(t), & t \geq T, \quad t \neq t_{k} .
\end{array}
$$

Choose a sequence $\left\{c_{n}\right\}$ such that $x\left(c_{n}\right)=0, T<c_{1}<\cdots<c_{n} \rightarrow+\infty, n \rightarrow+\infty$, $x(t) \geq 0$, for $t \in\left(c_{2 i-1}, c_{2 i}\right)$ and $x(t) \leq 0$ for $t \in\left(c_{2 i}, c_{2 i+1}\right)$. Let

$$
\hat{x}_{i}=\sup _{t \in\left(c_{2 i-1}, c_{21}\right)} x(t) \quad \text { and } \quad \tilde{x}_{i}=\inf _{t \in\left(c_{2 i}, c_{2 i+1}\right)} x(t) .
$$

Without loss of generality, we assume that $\lim \sup _{i \rightarrow \infty} \hat{x}_{i}=v$ and $\liminf _{i \rightarrow \infty} \tilde{x}_{i}=u$. First, we prove that

$$
\hat{x}_{i} \leq p\left(1-e^{u_{1}}\right)(1+\epsilon)
$$

or

$$
\hat{x}_{i} \leq p\left(1-e^{u_{1}}\right)(1+\epsilon)^{2} .
$$


There are two possible cases to consider.

Case 1: $\hat{x}_{i}$ is the maximum value of $x(t)$ in $\left(c_{2 i-1}, c_{2 i}\right)$. Then there exists $c \epsilon$ $\left(c_{2 i-1}, c_{2 i}\right)$ such that $\hat{x}_{i}=x(c)>0, x^{\prime}(c) \geq 0$. By $(1.1), x(c-\tau) \leq 0$, so there exists $\xi \in(c-\tau, c)$ such that $x(\xi)=0$, if $t \in[\xi, c]$ then $t-\tau \leq \xi$. Integrating (3.2) from $t-\tau$ to $\xi$, we have

$$
-\prod_{t-\tau \leq t_{k}<\xi}\left(1+b_{k}\right) x(t-\tau) e^{\int_{0}^{\prime-\tau} a(u) d u} \leq\left(1-e^{u_{1}}\right) \int_{t-\tau}^{\xi} p(s) e^{\int_{0} a(u) d u} \prod_{s \leq t_{k}<\xi}\left(1+b_{k}\right) d s .
$$

Since $1-e^{x} \leq-x$ and by (1.1), we have

$$
x^{\prime}(t)+a(t) x(t) \leq-p(t) x(t-\tau), \quad t \geq 0, t \neq t_{k} .
$$

Then

$$
x^{\prime}(t)+a(t) x(t) \leq\left(1-e^{u_{1}}\right) p(t) \int_{t-\tau}^{\xi} p(s) e^{\int_{t-\tau}^{s} a(u) d u} \prod_{t-\tau \leq t_{k}<s}\left(1+b_{k}\right)^{-1} d s .
$$

Integrating (3.8) from $\xi$ to $c$, we get

$$
\begin{aligned}
& x(c) e^{\int_{0}^{c} a(u) d u} \leq\left(1-e^{u_{1}}\right) \int_{\xi}^{c} p(t) e^{\int_{0}^{\prime} a(u) d u} \prod_{t \leq t_{k}<c}\left(1+b_{k}\right) \int_{t-\tau}^{\xi} p(s) e^{f_{1-\tau}^{s} a(u) d u} \\
& \times \prod_{1-t \leq t_{k}<s}\left(1+b_{k}\right)^{-1} d s d t \\
& \leq\left(1-e^{u_{1}}\right)\left[\int_{\xi}^{c} p(t) e^{\int_{0}^{t} a(u) d u} \prod_{t \leq t_{k}<c}\left(1+b_{k}\right)\left(p+\frac{1}{2} e^{-\int_{t--}^{\prime} a(u) d u}\right)\right. \\
& \times e^{\int_{t-\mathrm{r}}^{\prime} a(u) d u} d t-\int_{\xi}^{c} p(t) e^{\int_{0}^{\prime} a(u) d u} \prod_{t \leq t_{k}<c}\left(1+b_{k}\right) \int_{\xi}^{t} p(s) e^{\int_{0}^{s} a(u) d u} \\
& \left.\times \prod_{s \leq t_{k}<c}\left(1+b_{k}\right) d s e^{-f_{0}^{\prime-\tau} a(u) d u} \prod_{t-\tau \leq t_{k}<c}\left(1+b_{k}\right)^{-1} d t\right] .
\end{aligned}
$$

Using (2.2), (2.4) and (2.5), we obtain

$$
\begin{aligned}
x(c) e^{f_{0}^{c} a(u) d u} \leq & \left(1-e^{u_{1}}\right) \int_{\xi}^{c} p(t) e^{\int_{0}^{\prime} a(u) d u} \prod_{t \leq u_{k}<c}\left(1+b_{k}\right) d t\left(\frac{1}{2}+p e^{\int_{c-r}^{c} a(u) d u}\right) \\
& -\frac{1-e^{u_{1}}}{1+\epsilon} e^{-\int_{0}^{c-\tau} a(u) d u} \int_{\xi}^{c} p(t) e^{\int_{0}^{\prime} a(u) d u} \\
& \times \prod_{i \leq t_{k}<c}\left(1+b_{k}\right) \int_{\xi}^{t} p(s) e^{\int_{0}^{s} a(u) d u} \prod_{s \leq k_{k}<c}\left(1+b_{k}\right) d s d t
\end{aligned}
$$




$$
\begin{aligned}
= & \left(1-e^{u_{1}}\right) \int_{\xi}^{c} p(t) e^{\int_{0}^{\prime} a(u) d u} \prod_{t \leq t_{k}<c}\left(1+b_{k}\right) d t\left(\frac{1}{2}+p e^{\int_{r-\tau}^{c} a(u) d u}\right) \\
& -\frac{1-e^{u_{1}}}{1+\epsilon} e^{-\int_{0}^{c-t} a(u) d u} \frac{1}{2}\left(\int_{\xi}^{c} p(t) e^{\int_{0}^{t} a(u) d u} \prod_{t \leq t_{k}<c}\left(1+b_{k}\right) d t\right)^{2} .
\end{aligned}
$$

In the following, we consider two possible subcases.

Subcase 1.1: $\int_{\xi}^{c} p(t) e^{\int_{0}^{\prime} a(u) d u} \prod_{t \leq t_{k}<c}\left(1+b_{k}\right) d t \leq(1+\epsilon) e^{\int_{0}^{c-t} a(u) d u}$. Since the function

$$
\left(1 / 2+p e^{\int_{c-r}^{c} a(u) d u}\right) x-(1+\epsilon)^{-1} e^{-\int_{0}^{c-r} a(u) d u} x^{2} / 2
$$

is increasing, we obtain $x(c) e^{\int_{0}^{c} a(u) d u} \leq p\left(1-e^{u_{1}}\right) e^{\int_{0}^{c} a(u) d u}(1+\epsilon)$. Then (3.4) holds.

Subcase 1.2: $\int_{\xi}^{c} p(t) e^{\int_{0}^{\prime} a(u) d u} \prod_{t \leq t_{k}<c}\left(1+b_{k}\right) d t>(1+\epsilon) e^{\int_{0}^{c-\tau} a(u) d u}$. We choose $\eta \in(\xi, c)$ such that

$$
\int_{\eta}^{c} p(t) e^{\int_{0}^{t} a(u) d u} \prod_{t \leq t_{k}<c}\left(1+b_{k}\right) d t=(1+\epsilon) e^{\int_{0}^{c-t} a(u) d u} .
$$

Integrating (3.2) from $\xi$ to $\eta$, we have

$$
x(\eta) e^{\int_{0}^{\eta} a(u) d u} \leq\left(1-e^{u_{1}}\right) \int_{\xi}^{\eta} p(t) e^{\int_{0}^{\prime} a(u) d u} \prod_{t \leq t_{k}<\eta}\left(1+b_{k}\right) d t .
$$

Integrating (3.8) from $\eta$ to $c$,

$$
\begin{aligned}
x(c) e^{\int_{0}^{c} a(u) d u}- & \prod_{\eta \leq t_{k}<c}\left(1+b_{k}\right) x(\eta) e^{\int_{0}^{\eta} a(u) d u} \\
\leq & \left(1-e^{u_{1}}\right) \int_{\eta}^{c} p(t) e^{\int_{0}^{t} a(u) d u} \prod_{t \leq t_{k}<c}\left(1+b_{k}\right) \int_{t-\tau}^{\xi} p(s) e^{\int_{t-\tau}^{\prime} a(u) d u} \\
& \times \prod_{t-\tau \leq t_{k}<c}\left(1+b_{k}\right)^{-1} d s d t .
\end{aligned}
$$

Then we get

$$
\begin{aligned}
x(c) e^{\int_{0}^{c} a(u) d u} \leq & \left(1-e^{u_{1}}\right)\left[\int_{\xi}^{\eta} p(t) e^{\int_{0}^{\prime} a(u) d u} \prod_{t-\tau \leq t_{k}<c}\left(1+b_{k}\right) d t\right. \\
& +\int_{\eta}^{c} p(t) e^{\int_{0}^{t} a(u) d u} \prod_{t \leq t_{k}<c}\left(1+b_{k}\right) \int_{t-\tau}^{\xi} p(s) e^{\int_{i-\tau}^{s} a(u) d u} \\
& \left.\times \prod_{t-\tau \leq t_{k}<s}\left(1+b_{k}\right)^{-1} d s d t\right]
\end{aligned}
$$


Similarly to the argument we used in Subcase 1.1, we get

$$
\begin{aligned}
x(c) e^{\int_{0}^{c} a(u) d u} \leq & \left(1-e^{u_{1}}\right) \int_{\xi}^{\eta} p(t) e^{\int_{0}^{t} a(u) d u} \prod_{t \leq t_{k}<c}\left(1+b_{k}\right) d t \\
& -\frac{1-e^{u_{1}}}{2(1+\epsilon) e^{\int_{0}^{c-\tau} a(u) d u}}\left[\left(\int_{\xi}^{c} p(t) e^{\int_{0}^{\prime} a(u) d u} \prod_{t \leq t_{k}<c}\left(1+b_{k}\right) d t\right)^{2}\right. \\
& \left.-\left(\int_{\xi}^{\eta} p(t) e^{\int_{0}^{t} a(u) d u} \prod_{t-r \leq t_{k}<c}\left(1+b_{k}\right) d t\right)^{2}\right] \\
& +\left(1-e^{u_{1}}\right)\left(\frac{1}{2}+p e^{\int_{c-r}^{c} a(u) d u}\right) \int_{\eta}^{c} p(t) e^{\int_{0}^{t} a(u) d u} \prod_{t \leq t_{k}<c}\left(1+b_{k}\right) d t \\
= & p\left(1-e^{u_{1}}\right)(1+\epsilon) e^{\int_{0}^{c} a(u) d u} .
\end{aligned}
$$

Hence (3.4) is proved.

Case 2: $\hat{x}_{i}$ is not the maximum value of $x(t)$ in $\left(c_{2 i-1}, c_{2 i}\right)$. Then there exists $t_{k+l} \in\left(c_{2 i-1}, c_{2 i}\right)$ such that $\hat{x}_{i}=x\left(t_{k+l}^{+}\right)$. Suppose that $c_{2 i-1}<t_{k+1}<\cdots<t_{k+l}$. Proving that $x(t)$ is bounded, we obtain

$$
\hat{x}_{i} \leq \prod_{s=j}^{l}\left(1+b_{k}\right) p\left(1-e^{u_{1}}\right)(1+\epsilon), \quad j=1,2, \ldots, l .
$$

Then $\hat{x}_{i} \leq(1+\epsilon)^{2} p\left(1-e^{u_{1}}\right)$. From (3.4) and (3.5), let $i \rightarrow+\infty$ and $\epsilon \rightarrow 0$ to obtain

$$
v \leq p\left(1-e^{u}\right)
$$

Next, we shall prove

$$
u \geq(p+1 / 2)\left(1-e^{v}\right)
$$

There are two cases to consider.

Case 1: $\tilde{x}_{i}$ is the minimum value of $x(t)$ in $\left[c_{2 i}, c_{2 i+1}\right]$. Then there exists $c \in$ $\left(c_{2 i}, c_{2 i+1}\right)$ such that $x(c)=\tilde{x}_{i} \leq 0, x^{\prime}(c)=0$. By $(1.1), x(c-\tau) \geq 0$. Then there exists $\xi \in[c-\tau, c)$ such that $x(\xi)=0$. Integrating (3.3) from $\xi$ to $c$, we obtain

$$
x(c) e^{\int_{0}^{c} a(u) d u} \geq\left(1-e^{v_{1}}\right) \int_{\xi}^{c} p(t) e^{\int_{0}^{t} a(u) d u} \prod_{t \leq t_{k}<c}\left(1+b_{k}\right) d t .
$$

Then by (2.2) and (2.4), we get

$$
\tilde{x}_{i} \geq(1+\epsilon)\left(1-e^{v_{1}}\right)(p+1 / 2) .
$$


Case 2: $\tilde{x}_{i}$ is not the minimum value of $x(t)$ in $\left(c_{2 i}, c_{2 i+1}\right)$. Then there exists $t_{k+l} \in\left(c_{2 i}, c_{2 i+1}\right)$ such that $\tilde{x}_{i}=x\left(t_{k+l}^{+}\right)$. Suppose that $c_{2 i}<t_{k+1}<\cdots<t_{k+l}$. Proving that $x(t)$ is bounded, we get

$$
\tilde{x}_{i} \geq \prod_{s=j}^{l}\left(1+b_{k+s}\right)(1+\epsilon)(p+1 / 2)\left(1-e^{v_{1}}\right) .
$$

By (2.2), $\tilde{x}_{i} \geq(1+\epsilon)^{2}(p+1 / 2)\left(1-e^{v_{1}}\right)$. Let $i \rightarrow+\infty$ and $\epsilon \rightarrow 0$. By (3.11) and (3.12), we get (3.10). From (2.1), (3.9), (3.10) and the fact that $-\infty<u \leq 0 \leq v<$ $+\infty$, we get $u=v=0$. Then $x(t)$ tends to zero as $t \rightarrow \infty$. By Lemmas 1 and 3, Theorem 1 is proved.

In order to prove Theorem 2, we need the following lemma.

LEMMA 4. Suppose that (2.2), (2.5) and (2.6) hold. Then every oscillatory solution of (1.1) tends to zero as $t \rightarrow \infty$.

Proof. From Lemma 2, $x(t)$ is bounded. By the proof of Lemma 3, we get (3.2), (3.3) and (3.6). Choose $\left\{c_{n}\right\}$ satisfing the conditions in Lemma 3, with $\hat{x}_{i} \rightarrow v, \tilde{x}_{i} \rightarrow u$ as $i \rightarrow+\infty$. There are two cases to consider.

Case 1: $\hat{x}_{i}$ is the maximum value of $x(t)$ in $\left(c_{2 i-1}, c_{2 i}\right)$. Substituting (3.6) into (1.1), we have, for $t \in[\xi, c], t \neq t_{k}$,

$$
\begin{aligned}
x^{\prime}(t) & +a(t) x(t) \\
& \leq p(t)\left[1-\exp \left(-A \int_{t-\tau}^{\xi} p(s) e^{\int_{t-\tau}^{s} a(u) d u} \prod_{t-\tau \leq t_{k}<s}\left(1+b_{k}\right)^{-1} d s\right)\right],
\end{aligned}
$$

where $1-e^{u_{1}}=A$. Integrating (3.13) from $\xi$ to $c$, we get

$$
\begin{aligned}
x(c) e^{\int_{0}^{c} a(u) d u} \leq & \int_{\xi}^{c} p(t)\left[1-\exp \left(-A \int_{t-\tau}^{\xi} p(s) e^{\int_{t-\tau}^{s} a(u) d u} \prod_{t-\tau \leq t_{k}<s}\left(1+b_{k}\right)^{-1} d s\right)\right] \\
& \times e^{\int_{0}^{t} a(u) d u} \prod_{t \leq t_{k}<c}\left(1+b_{k}\right) d t \\
\leq & \int_{\xi}^{c} p(t) e^{\int_{0}^{t} a(u) d u} \prod_{t \leq t_{k}<c}\left(1+b_{k}\right) d t-\int_{\xi}^{c} p(t) e^{\int_{0}^{t} a(u) d u} \\
& \times \prod_{t \leq t_{k}<c}\left(1+b_{k}\right) \exp \left(-A \int_{t-\tau}^{t} p(s) e^{\int_{t-\tau}^{t} a(u) d u} \prod_{t-\tau \leq t_{k}<s}\left(1+b_{k}\right)^{-1} d s\right. \\
& \left.+A \int_{\xi}^{t} p(s) e^{\int_{t-\tau}^{s} a(u) d u} \prod_{t-\tau \leq t_{k}<s}\left(1+b_{k}\right)^{-1} d s\right) d t
\end{aligned}
$$




$$
\begin{aligned}
\leq & \int_{\xi}^{c} p(t) e^{\int_{0}^{t} a(u) d u} \prod_{t \leq t_{k}<c}\left(1+b_{k}\right) d t \\
& -e^{-3 A / 2} \int_{\xi}^{c} p(t) e^{\int_{0}^{\prime} a(u) d u} \prod_{t \leq t_{k}<c}\left(1+b_{k}\right) \\
& \times \exp \left(\frac{A \int_{\xi}^{t} p(s) e^{\int_{0}^{s} a(u) d u} \prod_{s \leq t_{k}<c}\left(1+b_{k}\right) d s}{e^{\int_{0}^{c-r} a(u) d u} \prod_{t-\tau \leq t_{k}<c}\left(1+b_{k}\right)}\right) d t \\
\leq & \int_{\xi}^{c} p(t) e^{\int_{0}^{t} a(u) d u} \prod_{t \leq t_{k}<c}\left(1+b_{k}\right) d t-e^{-3 A / 2} A^{-1}(1+\epsilon) e^{\int_{0}^{c-\tau} a(u) d u} \\
& \times\left[\exp \left(\frac{A \int_{\xi}^{c} p(s) e^{\int_{0}^{s} a(u) d u} \prod_{s \leq t_{k}<c}\left(1+b_{k}\right) d s}{(1+\epsilon) e^{\int_{0}^{c-t} a(u) d u}}\right)-1\right] .
\end{aligned}
$$
Then

Case 1.1: $\int_{\xi}^{c} p(t) e^{\int_{0}^{\prime} a(u) d u} \prod_{t \leq t_{k}<c}\left(1+b_{k}\right) d t \leq-(1 / A) \ln (1-A) e^{\int_{0}^{c-\tau} a(u) d u}(1+\epsilon)$.

$$
\begin{aligned}
x(c) e^{\int_{0}^{c} a(u) d u} & \leq-\frac{\ln (1-A)}{A} e^{\int_{0}^{c-\tau} a(u) d u}(1+\epsilon)-\frac{e^{-3 A / 2}}{(1-A)(1+\epsilon)^{-1} e^{-\int_{0}^{c-\tau} a(u) d u}} \\
& =-\frac{\ln (1-A)}{A} e^{\int_{0}^{c-\tau} a(u) d u}(1+\epsilon)-\frac{1+\epsilon}{1-A} e^{-3 A / 2} e^{\int_{0}^{c-\tau} a(u) d u},
\end{aligned}
$$

so

$$
x(c) \leq(1+\epsilon)\left(-\frac{\ln (1-A)}{A}-\frac{e^{-3 A / 2}}{1-A}\right) .
$$

By Kuang's method [1, (2.21)], we get

$$
\hat{x}_{i}=x(c) \leq(1+\epsilon)\left(A-A^{2} / 6\right)
$$

Case 1.2:

$$
\begin{aligned}
\int_{\xi}^{c} p(t) e^{\int_{0}^{t} a(u) d u} \prod_{t \leq t_{k}<c}\left(1+b_{k}\right) d t & \leq \frac{3}{2} e^{\int_{0}^{c-\tau} a(u) d u}(1+\epsilon) \\
& <-\frac{\ln (1-A)}{A} e^{\int_{0}^{c-t} a(u) d u}(1+\epsilon) .
\end{aligned}
$$

Then, integrating (3.13) from $\xi$ to $c$, similarly to Case 1.1 , we get

$$
\hat{x}_{i}=x(c) \leq 3(1+\epsilon) / 2+(1+\epsilon)\left(e^{3 A / 2}-1\right) / A .
$$

By a method similar to that used by Kuang in [1, (2.19)], we get (3.14).

Case 1.3: $\int_{\xi}^{c} p(t) e^{\int_{0}^{t} a(u) d u} \prod_{t \leq l_{k}<c}\left(1+b_{k}\right) d t>-(1 / A) \ln (1-A)(1+\epsilon) e^{\int_{0}^{c-t} a(u) d u}$. Choose $\eta \in(\xi, c)$ such that

$$
\int_{\eta}^{c} p(t) e^{\int_{0}^{t} a(u) d u} \prod_{t \leq t_{k}<c}\left(1+b_{k}\right) d t=-\frac{\ln (1-A)}{A}(1+\epsilon) e^{\int_{0}^{c-\tau} a(u) d u} .
$$


Integrating (3.2) from $\xi$ to $\eta$, we have

$$
x(\eta) e^{\int_{0}^{\eta} a(u) d u} \leq A \int_{\xi}^{\eta} p(t) e^{\int_{0}^{\eta} a(u) d u} \prod_{t \leq t_{k}<\eta}\left(1+b_{k}\right) d t .
$$

Integrating (3.13) from $\eta$ to $c$, we have

$$
\begin{aligned}
& x(c) e^{\int_{0}^{c} a(u) d u}-\prod_{\eta \leq t_{k}<c}\left(1+b_{k}\right) x(\eta) e^{\int_{0}^{\eta} a(u) d u} \\
& \leq \int_{\eta}^{c} p(t) e^{\int_{0}^{\prime} a(u) d u} \prod_{t \leq t_{k}<c}\left(1+b_{k}\right)\left[1-\exp \left(-A \int_{t-\tau}^{\xi} \frac{p(s) e^{\int_{t-\tau}^{s} a(u) d u}}{\prod_{t-\tau \leq t_{k}<s}\left(1+b_{k}\right)} d s\right)\right] d t .
\end{aligned}
$$

Deleting $x(\eta)$, we obtain

$$
\begin{aligned}
x(c) e^{\int_{0}^{c} a(u) d u} & \leq A \int_{\xi}^{\eta} p(t) e^{\int_{0}^{t} a(u) d u} \prod_{t \leq t_{k}<c}\left(1+b_{k}\right) d t+\int_{\eta}^{c} p(t) e^{\int_{0}^{\prime} a(u) d u} \prod_{t \leq t_{k}<c}\left(1+b_{k}\right) d t \\
& -e^{-3 A / 2} \int_{\eta}^{c} p(t) e^{\int_{0}^{t} a(u) d u} \prod_{t \leq t_{k}<c}\left(1+b_{k}\right) \exp \left(A \int_{\xi}^{t} \frac{p(s) e^{\int_{t-\tau}^{3} a(u) d u}}{\prod_{t-\tau \leq t_{k}<s}\left(1+b_{k}\right)} d s\right) d t \\
\leq & A \int_{\xi}^{\eta} p(t) e^{\int_{0}^{t} a(u) d u} \prod_{t \leq t_{k}<c}\left(1+b_{k}\right) d t+\int_{\eta}^{c} p(t) e^{\int_{0}^{t} a(u) d u} \prod_{t \leq t_{k}<c}\left(1+b_{k}\right) d t \\
& -\frac{1+\epsilon}{e^{3 A / 2} A} e^{\int_{0}^{c-t} a(u) d u}\left[\exp \left(\frac{A}{1+\epsilon} \int_{\xi}^{c} p(t) e^{\int_{c-\tau}^{t} a(u) d u} \prod_{i \leq t_{k}<c}\left(1+b_{k}\right) d t\right)\right. \\
& \left.-\exp \left(\frac{A}{1+\epsilon} \int_{\xi}^{\eta} p(t) e^{\int_{c-\tau}^{t} a(u) d u} \prod_{t \leq t_{k}<c}\left(1+b_{k}\right) d t\right)\right] .
\end{aligned}
$$

Then

$$
\begin{aligned}
\hat{x}_{i}=x(c) \leq & A(1+\epsilon) e^{-\int_{c-\tau}^{c} a(u) d u} \int_{\xi}^{\eta} p(t) e^{\int_{c-t}^{t} a(u) d u} \prod_{t \leq t_{k}<c}\left(1+b_{k}\right) d t \\
& +e^{-\int_{c-\tau}^{c} a(u) d u} \int_{\eta}^{c} p(t) e^{\int_{c-\tau}^{t} a(u) d u} \prod_{t \leq t-k<c}\left(1+b_{k}\right) d t \\
& -\frac{1+\epsilon}{e^{3 A / 2} A} e^{-\int_{c-\tau}^{c} a(u) d u}\left(\exp \left(\frac{A}{1+\epsilon} \int_{\xi}^{c} p(t) e^{\int_{c-\tau}^{\prime} a(u) d u} \prod_{t \leq t_{k}<c}\left(1+b_{k}\right) d t\right)\right. \\
& \left.-\exp \left(\frac{A}{1+\epsilon} \int_{\xi}^{\eta} p(t) e^{\int_{c-\tau}^{\prime} a(u) d u} \prod_{t \leq t_{k}<c}\left(1+b_{k}\right) d t\right)\right)
\end{aligned}
$$




$$
\leq(1+\epsilon)(3 A / 2-((1-A) / A) \ln (1-A)-1) \text {. }
$$

By Kuang's method in [1, (2.21)], we get (3.14).

Case 2: If $\hat{x}_{i}$ is not the maximum value of $x(t)$ in $\left(c_{2 i-1}, c_{2 i}\right)$, then there exists $t_{k+l} \in\left(c_{2 i-1}, c_{2 i}\right)$ such that $\hat{x}_{i}=x\left(t_{k+l}\right)$. Suppose that $c_{2 i-1}<t_{k+1}<\cdots<t_{k+l}$. Then we can obtain

$$
\hat{x}_{i} \leq \prod_{s=j}^{l}\left(1+b_{k+s}\right)(1+\epsilon)\left(A-A^{2} / 6\right), \quad j=1,2, \ldots, l .
$$

Then by (2.2), we get $\hat{x}_{i} \leq(1+\epsilon)^{2}\left(A-A^{2} / 6\right)$. By (3.13) and (3.14), let $i \rightarrow+\infty$ and $\epsilon \rightarrow 0$ to obtain $v \leq\left(1-e^{u}\right)-\left(1-e^{u}\right)^{2} / 6$.

Next we prove

$$
u \geq\left(1-e^{v}\right)-\left(1-e^{v}\right)^{2} / 6
$$

There are two cases to consider.

Case 1: $\tilde{x}_{i}$ is the minimum value of $x(t)$ in $\left(c_{2 i}, c_{2 i+1}\right)$. Then there exists $c \in$ $\left(c_{2 i}, c_{2 i+1}\right)$ such that $x(c)=\tilde{x}_{i}<0, x^{\prime}(c) \leq 0$. There exists $\xi \in(c-\tau, c)$ such that $x(\xi)=0$. If $t \in[\xi, c]$, then $t-\tau \leq \xi$. Integrating (3.3) from $t-\tau$ to $\xi$, then

$$
-\prod_{t-\tau \leq t_{k}<\xi}\left(1+b_{k}\right) x(t-\tau) e^{f_{0}^{t-\tau} a(u) d u} \geq B \int_{t-\tau}^{\xi} p(s) e^{f_{t-\tau}^{s} a(u) d u} \prod_{t-\tau \leq t_{k}<s}\left(1+b_{k}\right) d s,
$$

where $B=1-e^{v_{1}}$. By (1.1), we get, for $t \in[\xi, c], t \neq t_{k}$,

$$
x^{\prime}(t)+a(t) x(t) \geq p(t)\left(1-\exp \left(-B \int_{t-\tau}^{\xi} \frac{p(s) e^{\int_{t-\tau}^{3} a(u) d u}}{\prod_{t-\tau \leq t_{k}<s}\left(1+b_{k}\right)} d s\right)\right) .
$$

There are three subcases to consider.

Subcase 1.1: $\int_{\xi}^{c} p(t) e^{\int_{t-r}^{t} a(u) d u} \prod_{t \leq t_{k}<c}\left(1+b_{k}\right) d t \leq 1+\epsilon$. Integrating (3.7) from $\xi$ to $c$, we have

$$
\tilde{x}_{i}=x(c) \geq B \int_{\xi}^{c} p(t) e^{\int_{c}^{t} a(u) d u} \prod_{t \leq t_{k}<c}\left(1+b_{k}\right) d t
$$

Then

$$
\bar{x}_{i} \geq(1+\epsilon) B \geq(1+\epsilon)\left(B-B^{2} / 6\right)
$$

Subcase 1.2:

$$
1+\epsilon<\int_{\xi}^{c} p(t) e^{\int_{t-\mathrm{r}}^{t} a(u) d u} \prod_{t \leq t_{k}<c}\left(1+b_{k}\right) d t \leq\left(\frac{3}{2}+\frac{\ln (1-B)}{B}\right)(1+\epsilon) .
$$


Integrating (3.3) from $\xi$ to $c$, we have

$$
\begin{aligned}
\tilde{x}_{i}=x(c) & \geq B \int_{\xi}^{c} p(t) e^{\int_{c}^{\prime} a(u) d u} \prod_{t \leq t_{k}<c}\left(1+b_{k}\right) d t \\
& \geq B\left(\frac{3}{2}+\frac{\ln (1-B)}{B}\right)(1+\epsilon) \geq(1+\epsilon)\left(B-\frac{1}{6} B^{2}\right) .
\end{aligned}
$$

Hence (3.17) is proved.

Subcase 1.3:

$$
\frac{3}{2}(1+\epsilon) \geq \int_{\xi}^{c} p(t) e^{\int_{c-t}^{t} a(u) d u} \prod_{t \leq t_{k}<c}\left(1+b_{k}\right) d t>\left(\frac{3}{2}+\frac{\ln (1-B)}{B}\right)(1+\epsilon) .
$$

Choose $\eta \in(\xi, c)$ such that

$$
\int_{\eta}^{c} p(t) e^{\int_{c-\gamma}^{t} a(u) d u} \prod_{t \leq t_{k}<c}\left(1+b_{k}\right) d t=\left(\frac{3}{2}+\frac{\ln (1-B)}{B}\right)(1+\epsilon) .
$$

Integrating (3.3) from $\xi$ to $\eta$ and integrating (3.16) from $\eta$ to $c$, we obtain

$$
\begin{aligned}
& \tilde{x}_{i}=x(c) \\
& \geq B \int_{\xi}^{\eta} p(t) e^{\int_{c}^{\prime} a(u) d u} \prod_{i \leq t_{k}<c}\left(1+b_{k}\right) d t+\int_{\eta}^{c} p(t) e^{\int_{r}^{t} a(u) d u} \prod_{i \leq i_{k}<c}\left(1+b_{k}\right) d t \\
& -e^{-3 B / 2} \int_{\eta}^{c} p(t) e^{\int_{c-r}^{\prime} a(u) d u} \prod_{t \leq t_{k}<c}\left(1+b_{k}\right) \\
& \times \exp \left(\frac{B}{1+\epsilon} \int_{\xi}^{t} p(s) e^{\int_{c-s}^{t} a(u) d u} \prod_{s \leq t_{k}<c}\left(1+b_{k}\right) d s\right) d t \\
& \geq B \int_{\xi}^{\eta} p(t) e^{\int_{c-\tau}^{t} a(u) d u} \prod_{t \leq I_{k}<c}\left(1+b_{k}\right) d t+\int_{\eta}^{c} p(t) e^{\int_{r-\tau}^{t} a(u) d u} \prod_{t \leq t_{k}<c}\left(1+b_{k}\right) d t \\
& -\frac{1+\epsilon}{B e^{3 B / 2}}\left(\exp \left(\frac{B}{1+\epsilon} \int_{\xi}^{c} p(t) e^{\int_{c-t}^{t} a(u) d u} \prod_{t \leq t_{k}<c}\left(1+b_{k}\right) d t\right)\right. \\
& \left.-\exp \left(\frac{B}{1+\epsilon} \int_{\xi}^{\eta} p(t) e^{\int_{c-s}^{\prime} a(u) d u} \prod_{t \leq t_{k}<c}\left(1+b_{k}\right) d t\right)\right) \\
& \geq(1+\epsilon)\left(\frac{3}{2} B-\frac{1}{B}((1-B) \ln (1-B)+B)\right) \geq(1+\epsilon)\left(B-\frac{1}{6} B^{2}\right) \text {. }
\end{aligned}
$$

Then (3.17) is proved. The last inequality is obtained by the method used by Yu in [6, page 234]. 
Case 2: $\tilde{x}_{i}$ is not the minimum value of $x(t)$ in $\left(c_{2 i}, c_{2 i+1}\right)$. Then there exists $t_{k+l} \in\left(c_{2 i}, c_{2 i+1}\right)$ such that $\tilde{x}_{i}=x\left(t_{k+l}^{+}\right)$. Suppose that $c_{2 i}<t_{k+1}<\cdots<t_{k+l}$. Then we can obtain $\tilde{x}_{i} \geq \prod_{s=j}^{l}\left(1+b_{k+s}\right)(1+\epsilon)\left(B-B^{2} / 6\right), j=1,2, \ldots, l$. By (2.2), we have

$$
\tilde{x}_{i} \geq(1+\epsilon)^{2}\left(B-B^{2} / 6\right)
$$

From (3.17) and (3.18), let $i \rightarrow+\infty$ and $\epsilon \rightarrow 0$ to obtain (3.15).

Let $1-e^{u}=x, 1-e^{v}=-y$. Then (3.15) and (3.16) become

$$
\ln (1+y) \leq x-x^{2} / 6, \quad \ln (1-x) \geq-y-y^{2} / 6 \text {. }
$$

By [6, Lemma 1.4], $x=y=0$, so $u=v=0$. Then $x(t)$ tends to zero as $t \rightarrow \infty$. By Lemmas 1 and 4, we obtain Theorem 2 .

\section{Acknowledgements}

The authors wish to thank the referee for many concrete and helpful comments. This work was supported by the NNSF of China and the NSF of the Shandong Province of China.

\section{References}

[1] Y. Kuang, Delay differential equations with applications in population dynamics (Academic Press, Boston, 1993).

[2] V. Lakshmikantham, D. D. Bainov and P. S. Simenov, Theory of impulsive differential equations (World Scientific, Singapore, 1989).

[3] J. H. Shen, "Global existence and uniqueness, oscillation and nonoscillation of impulsive delay differential equations", Acta Math. Sinica 40 (1997) 53-59, (in Chinese).

[4] W. H. So Joseph and J. S. Yu, "Global attractivity for a population model with time delay", Proc. Amer. Math. Soc. 123 (1995) 2687-2694.

[5] M. Wazewska-Czyzewska and A. Lasota, "Mathematical problems of the dynamics of the red blood cells system", Ann. Polon. Math. 6 (1976) 23-40.

[6] J. S. Yu, "Global attractivity of zero solution of a class of delay differential equations and its applications", Sci. China Ser. A 39 (1996) 225-237.

[7] J. S. Yu, "Asymptotic stability of nonautonomous delay differential equations", Chinese Sci. Bull. 42 (1997) 1248-1252.

[8] J. S. Yu and B. G. Zhang, "Stability theorems for delay differential equations with impulses", $J$. Math. Anal. Appl. 199 (1996) 162-175.

[9] X. S. Zhang and J. Y. Yan, "Global attractivity in impulsive functional differential equations", Indian J. Pure Appl. Math. 29 (1998) 871-878. 